\title{
Spot Pricing of Secondary Spectrum Usage in Wireless Cellular Networks
}

\author{
Huseyin Mutlu, Murat Alanyali, and David Starobinski \\ Department of Electrical and Computer Engineering \\ Boston University, Boston, MA 02215 \\ E-mail: \{hmutlu,alanyali,staro\}@bu.edu
}

\begin{abstract}
Recent deregulation initiatives enable cellular providers to sell excess spectrum for secondary usage. In this paper, we investigate the problem of optimal spot pricing of spectrum by a provider in the presence of both non-elastic primary users, with long-term commitments, and opportunistic, elastic secondary users. We first show that optimal pricing can be formulated as an infinite horizon average reward problem and solved using stochastic dynamic programming. Next, we investigate the design of efficient single pricing policies. We provide numerical and analytical evidences that static pricing policies do not perform well in such settings (in sharp contrast to settings where all the users are elastic). On the other hand, we prove that deterministic threshold pricing achieves optimal profit amongst all single-price policies and performs close to global optimal pricing. We characterize the profit regions of static and threshold pricing, as a function of the arrival rate of primary users. Under certain reasonable assumptions on the demand function, we show that the profit region of threshold pricing can be far larger than that of static pricing. Moreover, we also show that these profit regions critically depend on the support of the demand function rather than specific form of it. We prove that the profit function of threshold pricing is unimodal in price and determine a restricted interval in which the optimal threshold lies. These two properties enable very efficient computation of the optimal threshold policy that is far faster than that of the global optimal policy.
\end{abstract}

\section{INTRODUCTION}

A major global effort is underway to deregulate wireless spectrum and achieve much better utilization of this scarce resource. The Secondary Markets Initiative [1] of the Federal Communications Commission (FCC), is one of the major steps towards achieving this goal. It permits leasing of spectrum licenses subject to approval by FCC. Similar regulatory efforts are also underway in the EU [2].

Consequences of the secondary markets initiative can already be felt with the emergence of secondary cellular providers, commonly called Mobile Virtual Network Operators (MVNOs) [3]. MVNOs buy spectrum and (possibly also infrastructure) from primary providers, referred to as Mobile Network Operators (MNOs). MVNOs add the value of better penetrating certain markets and offering differentiated products. A notable example of successful MVNO endeavor in the US is Virgin Mobile who has teamed up with Sprint Nextel as its MNO and recently reached a subscriber basis of over 4 millions customers [4].

This work was supported in part by the US National Science Foundation under grants CNS-0132802, CNS-0721860 and ANI-0238397
In this paper, we are interested in investigating how a provider, such as an MNO, should optimally price its excess spectrum to secondary users (SUs), such as MVNOs. On the one hand, a provider must ensure that the quality of service (QoS) of its primary users (PUs), who typically have longterm contracts, is not significantly affected by the admission of SUs. This is because the presence of SUs may increase the blocking of PU calls and hence lead to a punishment in the form of loss of business due to poor service. On the other hand, the provider is interested in maximizing its profit from the admission of SUs.

Given that the amount of excess spectrum is likely to fluctuate over time due to the inherent randomness in the PU traffic, spot pricing, based on real-time channel occupancy, emerges as the solution of choice. While spot and congestionbased pricing have been extensively studied in the literature (Cf. Section II), the typical model assumed in previous work differs significantly from the setting considered herein. Chiefly, most previous work assumes that the demand functions of all the users are elastic to price, i.e., all the arrival rates can be regulated with price. In contrast, in our setting, only the demand function of the SUs is elastic to price, but the arrival rate of PUs is not. As we will show, this difference is salient enough to result in fundamentally different structures for optimal (or near-optimal) pricing strategies.

Our first contribution in this paper is to formalize the profit maximization problem of a cellular provider in the presence of both PUs and SUs. Based on certain reasonable statistical assumptions, we show that optimal pricing can be formulated as an infinite horizon average reward problem and solved using stochastic dynamic programming.

Our second contribution is to investigate the design of efficient single pricing policies, i.e., policies where a provider can either admit a SU and charge a fixed price or reject a SU. These policies have the major advantage of making the cost of spectrum much more predictable to SUs. We first show that static pricing, which always applies the same admission price to SUs independently of the channel occupancy, may perform very poorly. This result stands in sharp contrast to the case where all the users are elastic to price. On the other hand, we provide numerical evidence that threshold pricing, which applies a fixed admission price to SUs when the channel occupancy is below some threshold $T$ and rejects them otherwise, performs very close to optimal. Further, we prove 
that among all the possible single-price admission policies (including randomized), threshold pricing is the optimal one.

Our third contribution is to characterize the profit regions of static pricing and threshold pricing. Our goal is to determine the maximum arrival rate of PUs, at which it is still possible to achieve profit from the admission of SUs. We characterize the profit region of static pricing and provide a lower bound (presumably tight) on the profit region of threshold pricing. We show that the profit region of threshold pricing is larger than that of static pricing. Through numerical example, we show that the difference can be quite large (e.g., three times larger). An interesting observation is that both the profit region of static pricing and the bound on threshold pricing depend only on the support of the demand function of the SUs, but not on its specific form. This result applies to quite general demand functions.

Our last contribution is to devise an efficient computational procedure to calculate the optimal threshold and price for threshold pricing. In particular, we prove that, for any given threshold $T$, the profit function is unimodal in price. This enables us to resort to well-known logarithmic search procedures to compute the optimal price. Moreover, we show that the optimal threshold is a non-decreasing function of price. By using this property, we are able to reduce the search interval for the optimal threshold, thus speeding up calculation of the optimal threshold policy. We also provide numerical results which show that the optimal threshold policy can be computed considerably faster than the global optimal policy.

The rest of the paper is organized as follows. In Section II, we survey related work. Our model and notation are introduced in Section III. In Section IV, we show how to derive the optimal pricing policy and present some characteristics of the optimal prices. In Section V, we investigate singleprice policies, prove the optimality of threshold pricing, and characterize the profit regions of static and threshold pricing. In Section $\mathrm{V}$, we also prove unimodality of profit function of threshold pricing. Then, in Section VI, we develop an efficient method to compute the optimal price and threshold for threshold pricing. We conclude the paper in Section VII.

\section{RELATED WORK}

The problem we consider of this paper is related to two well studied areas in communication networks, namely, pricing and call admission control. As such, we restrict our literature review to those papers that are the most relevant. A survey of other work related to pricing in cellular networks can be found in [5].

In [6], Paschalidis and Tsitsiklis investigate dynamic, congestion-based pricing of network resources. Their model assumes that all the users are elastic to price. They show that static pricing achieve good performance in general and can even be optimal in some asymptotic traffic regimes. This result was extended in [7] and [8], in the context of large network asymptotics. In [9], Ziya et. al. show that the optimal static price is unique. In [10], static spectrum pricing strategies capturing the effects of network-wide interferences are developed.

Threshold admission control policies have been extensively studied. Refs. [11,12] provide useful insights into the properties of such policies. The optimality of threshold pricing for certain optimization problem is proved in $[13,14]$. None of these papers integrate pricing into their formulations.

Refs. [15-17] integrate pricing with admission control in cellular networks. Ref. [15] considers time-of-day pricing methods. In our work, we consider pricing strategies that operate at much shorter time-scales, based on real-time information. Ref. [16] develops and evaluates "charge-by-time" pricing algorithms, while in our work we consider charging per admission. Ref. [17] develops a stochastic dynamic programming formulation that incorporates retrials. Our main contribution with respect to this previous body of work is to go beyond numerical optimizations and attempt to prove general structural properties, applicable to very general demand functions.

Ref. [18] analyzes a model similar to ours within the context of a generic rental management optimization problem. This work considers two type of customers, namely walkin and contract users. Walk-in users are priced according to congestion level of the system, similar to dynamic pricing of SUs in our model. Contract users, on the other hand, have fixed prices and arrival rates which are analogous to our PUs. Different than our work, [18] focuses on determining structures of the optimal policy rather than providing a simple, near-optimal alternative as done here.

\section{NETWORK MODEL}

In this section, we introduce our network model and notation (additional notation specific to static and threshold pricing will be provided in Section V). We consider a cellular network where each cell provides access to $C$ channels. In each cell, calls from PUs arrive according to a Poisson process with fixed rate $\lambda_{p}>0$. A punishment in the amount of $K$ monetary units is imposed if all the channels are busy and a PU call is blocked. SUs call arrivals also form a Poisson process that is independent of the PUs call arrivals process and its rate is modulated by the price charged by the provider. We thus assume that there is a demand function $\lambda_{s}(u)$ which determines the arrival rate of SU calls, where $u$ is the applied price. The price is a function of the state of the system, i.e., a SU pays a price $u_{n}$ for its call, if there are $n$ busy channels in the cell, where $0 \leq n<C$.

For both PUs and SUs, call holding times are exponentially distributed with rate $\mu$, independently of any other events. Without loss of generality, we will assume $\mu=1$, i.e., the mean call holding time is one unit of time.

The goal of the provider is to maximize the average profit per unit of time gained from accepting SUs. This quantity is denoted by $R$. We are interested in finding a pricing policy that satisfies this goal. A pricing policy is a rule that dictates which price should be advertised by the provider at any given point of time. 
Under the above assumptions, the system behavior follows the dynamics of a continuous-time birth-death Markov process, and explicit expression for the average profit $R$ can be provided as follows. First, let $\pi_{n}$ be the steady-state probability of finding the system in state $n$, i.e., there are $n$ busy channels. Next, let $\lambda_{n}=\lambda_{s}\left(u_{n}\right)+\lambda_{p}$ denote the total call arrival rate in state $n$ and $\Lambda=\left(\lambda_{0}, \lambda_{1}, \ldots, \lambda_{C-1}\right)$ denote the vector of arrival rates. Then, the probability of finding the system in state $n$, denoted by $\pi_{n}(\Lambda)$, can be explicitly written as follows:

$$
\pi_{n}(\Lambda)=\frac{\frac{\lambda_{0} \lambda_{1} \lambda_{2} \ldots \lambda_{n-1}}{n !}}{1+\frac{\lambda_{0}}{1 !}+\frac{\lambda_{0} \lambda_{1}}{2 !}+\ldots+\frac{\lambda_{0} \lambda_{1} \lambda_{2} \ldots \lambda_{C-1}}{C !}} .
$$

Due to the PASTA (Poisson Arrivals See Time Averages) property, the probability that a PU is blocked is $\pi_{C}(\Lambda)$. Thus, the average profit is

$$
R=\sum_{n=0}^{C-1} \pi_{n}(\Lambda) \lambda_{s}\left(u_{n}\right) u_{n}-\left(\pi_{C}(\Lambda)-E\left(\lambda_{p}, C\right)\right) \lambda_{p} K,
$$

where $E\left(\lambda_{p}, C\right)$ is the blocking probability of PUs in the absence of SU arrivals. This quantity corresponds to the wellknown Erlang-B formula

$$
E\left(\lambda_{p}, C\right)=\frac{\frac{\lambda_{p}^{C}}{C !}}{\sum_{n=0}^{C} \frac{\lambda_{p}^{n}}{n !}} .
$$

The first term in Eq. (2) represents the sum of the average revenues collected from SUs in each state. The second term is the average punishment due to accepted SUs. Note that, $\pi_{C}(\Lambda)-E\left(\lambda_{p}, C\right)$ corresponds to increase in blocking probability of PUs due to accepted SUs. $E\left(\lambda_{p}, C\right)$ acts as the normalization term to ensure that the profit is zero when all SUs are rejected.

In the sequel, we impose the following natural assumptions on the demand functions. These assumptions are required to guarantee the existence of a stationary optimal pricing policy and prove some of our structural results.

Assumption 3.1: There exists a price $u_{\max }$ for which $\lambda_{s}\left(u_{\max }\right)=0$. Moreover, $\lambda_{s}(u)$ is a strictly decreasing, differentiable function in $u$ over the interval $\left[0, u_{\max }\right]$ and $\lambda_{s}(0)$ is finite.

\section{Derivation of the Optimal Pricing Policy}

In this section, we derive the optimal pricing policy and present properties characterizing the optimal prices.

\section{A. Stochastic Dynamic Programming Formulation}

The maximization of the profit function in Eq. (2) is a complex multi-dimensional optimization problem and becomes quickly intractable as $C$ grows. One approach to alleviate this problem is to formulate it as an average reward stochastic dynamic programming (DP) problem [19,20]. Specifically, the optimal prices $u_{n}^{*}$ and optimal profit $R^{*}$ corresponding to the optimal policy can be computed using the so-called Bellman's equations since all the states in the Markov chain are recurrent (see Proposition 7.4.1 in [20]).

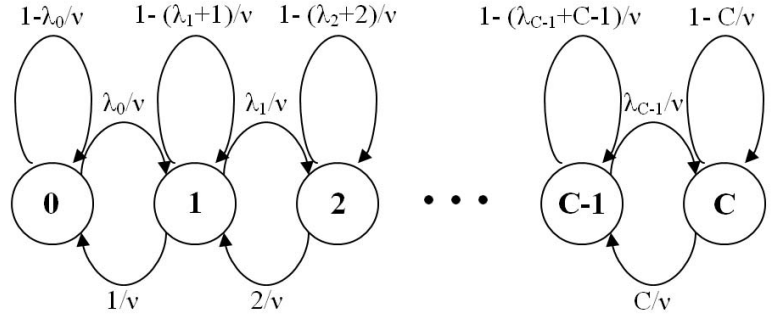

Fig. 1. Uniformized Markov Chain

Bellman's equations are usually formulated for discrete-time Markov chains. In our case, the Markov chain is continuous, but it can be discretized using a procedure called uniformization, where the transition rates out of each state are normalized by the maximum possible transition rate $v$, which in our case is given by the following expression:

$$
v=\lambda_{s}(0)+\lambda_{p}+C .
$$

The uniformized Markov chain with corresponding transition rates is shown in Fig. (1).

Bellman's equations are generally guaranteed to return the optimal solution only for finite action (control) space $\mathbb{U}$, where $\mathbb{U}$ represents the set of all possible prices advertised by the provider. Hence, prices must be discretized. We denote the discretization step with $\Delta u$. The cardinality of the action space is thus $|\mathbb{U}|=u_{\max } / \Delta u$. The resulting loss in profit due to discretization of price is bounded by $\lambda(0) \Delta u$, which can be made arbitrarily small at the expense of higher computational complexity (in Section VI, we describe an efficient computational procedure, applicable to threshold pricing, that scales to very large cardinality $|\mathbb{U}|)$.

Equipped with the above formulation, we can now compute the optimal pricing policy using the Bellman equations:

$$
\begin{aligned}
J^{*}+h(n)= & \max _{u_{n} \in \mathbb{U}}\left[\lambda_{s}\left(u_{n}\right) u_{n}+h(n+1) \frac{\lambda\left(u_{n}\right)}{v}\right. \\
& \left.+h(n-1) \frac{n}{v}+h(n)\left(1-\frac{\lambda\left(u_{n}\right)}{v}-\frac{n}{v}\right)\right]
\end{aligned}
$$

for $n=0,1,2 \ldots C-1$ and

$$
J^{*}=-\lambda_{p} K+h(C-1) \frac{C}{v},
$$

whereas the optimal profit is:

$$
R^{*}=J^{*}+E\left(\lambda_{p}, C\right) \lambda_{p} K .
$$

The first term in Eq. (5) represents the profit gained at state $n$ from the acceptance of a SU. The second and third terms are contributions to the revenue if the next transition is an arrival or departure, respectively. The last term is a consequence of the uniformization procedure. The effect of punishment due to blocked PU calls is captured by the first term in Eq. (6). The prices maximizing the RHS of Eq. (5) represent the optimal prices.

The unknowns in the above equations are $h(n)$ and $J^{*}$. The quantities $h(n)$ denote the relative reward in state $n$ with respect to state $C$. When the optimal policy is applied, $h(n) / v$ 


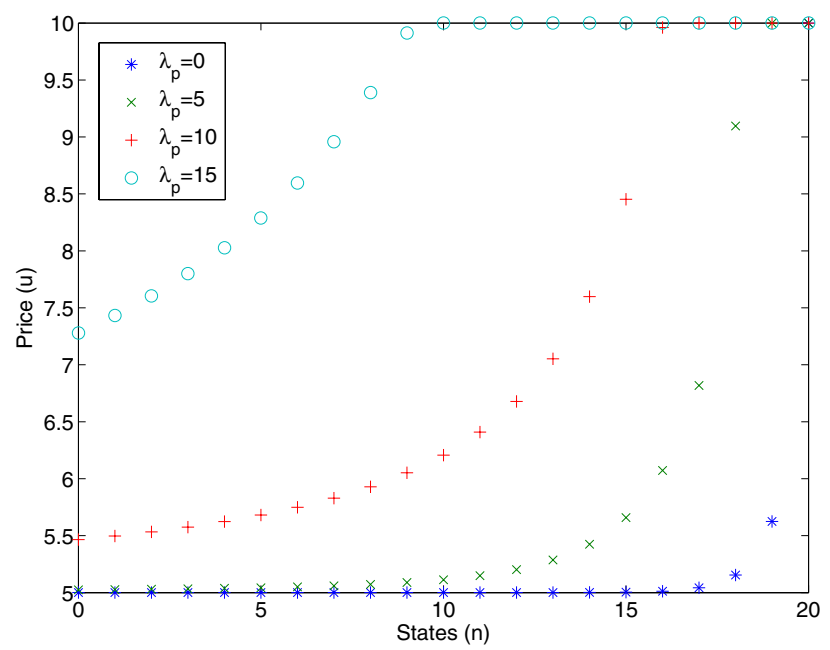

Fig. 2. Optimum prices for various PU arrival rates $\left(\lambda_{p}\right) . C=20, K=100$, $\lambda_{s}(u)=(10-u)+$ and $\Delta u=10^{-6}$

represents the difference between the total revenue gained over an infinite time horizon when starting the process from state $n$ and that gained when starting from state $C$. The quantities $R^{*}$ and $J^{*}$ differ only by a normalization constant used to ensure the non-negativity of the profit.

The solution of Bellman equation can be obtained by using various techniques described in the literature, such as policy iteration or relative value iteration $[19,20]$. Policy iteration theoretically requires on the order of $O\left(|\mathbb{U}|^{C}\right)$ iterations to converge while value iteration is not guaranteed to converge within a finite number steps. However, value iteration has a lower computational complexity at each iteration. In practice, as in other infinite horizon average reward problems [21], policy iteration appears to converge faster.

For different PU arrival rates $\lambda_{p}$, Figure (2) shows the values of the optimal prices (computed using policy iteration), for the demand function $\lambda_{s}(u)=(10-u)_{+}$(where $\left.(\cdot)_{+}=\max (\cdot, 0)\right)$, and parameters $C=20, K=100$, and $\Delta u=10^{-6}$. The figure indicates that, as $\lambda_{p}$ increases, the prices become higher in each state, and that SUs should not be accepted when the number of busy channels exceeds a certain threshold. More insight into this behavior will be provided in the sequel.

\section{B. Properties of the Optimal Policy}

In this section, we provide some results characterizing the optimal prices. First, we consider the ideal case of unlimited capacity.

Lemma 4.1: In the infinite capacity case (i.e., $C \rightarrow \infty$ ), the optimal price in each state is

$$
u_{\infty}=\arg \max _{u \in \mathbb{U}}\left(\lambda_{s}(u) u\right),
$$

and the corresponding profit is

$$
R_{\infty}=\lambda_{s}\left(u_{\infty}\right) u_{\infty}
$$

Note that $R_{\infty}$ is an upper bound on the profit achievable in any finite capacity system.

The following lemma states that in a finite capacity system, the optimal price in each state is larger than $u_{\infty}$.

Lemma 4.2: For any $0 \leq n \leq C-1, u_{n}^{*} \geq u_{\infty}$.

The next result states that the optimal prices are monotonically increasing in $n$.

Lemma 4.3: For any $0 \leq n \leq C-1, u_{n+1}^{*} \geq u_{n}^{*}$.

Proofs of these properties follow similar methods to those used in [6]. The main difference lies in taking into consideration the effects of PU arrivals and punishments. These proofs can be found in [22].

A consequence of the above properties is that the optimal price for any state lies between $u_{\infty}$ and $u_{\max }$. This fact can be exploited to reduce the size of the action space $\mathbb{U}$ when computing the optimal prices using Eq. (5).

\section{Single-Price Policies}

In this section, we investigate the design of single-price policies. In each state, these policies can either admit a SU and charge a fixed price $u$ or reject a SU (which is equivalent to ask for a price $u_{\max }$ or higher). For such policies the objective is to optimize the value of $u$ as well as the admission policy i.e., the decision of whether or not to admit a SU that is willing to pay the price. These policies are attractive because they allow a provider to advertise a single-price. They are also computationally easier to derive. Moreover, compared to optimal pricing, they provide more insight into the structure of good pricing policies.

A simple single-price policy is the so-called static pricing where SU calls are always applied the same admission price, unless all the channels are busy. For the cases where the demand functions of all the users are elastic to price and punishments are not imposed, static pricing is known to perform well and to be even asymptotically optimal in several regimes [6-8]. However, in this section, we show that, in the presence of inelastic users (PU) and punishments for blocked PU calls, the performance of static pricing degrades significantly.

Instead, we show next that among all single-price policies (including randomized), a deterministic threshold pricing policy performs optimally. In threshold pricing, SU calls are admitted and charged a price $u$ when the channel occupancy is smaller than some threshold $T$ and rejected otherwise. We provide numerical evidence showing that threshold pricing performs very close to optimal.

\section{A. Optimality of Threshold Pricing}

Theorem 5.1: For any price $u$ (including the optimal one), a threshold admission policy is optimal among all single-price policies.

Proof: Revenue-wise, gaining $u$ for each admitted SU call is the same as getting punished $u$ for each rejected one. Therefore, the problem can be reformulated as to minimize punishment. This problem is identical to the well-known MINOBJ problem analyzed in [13] where SU and PU calls 


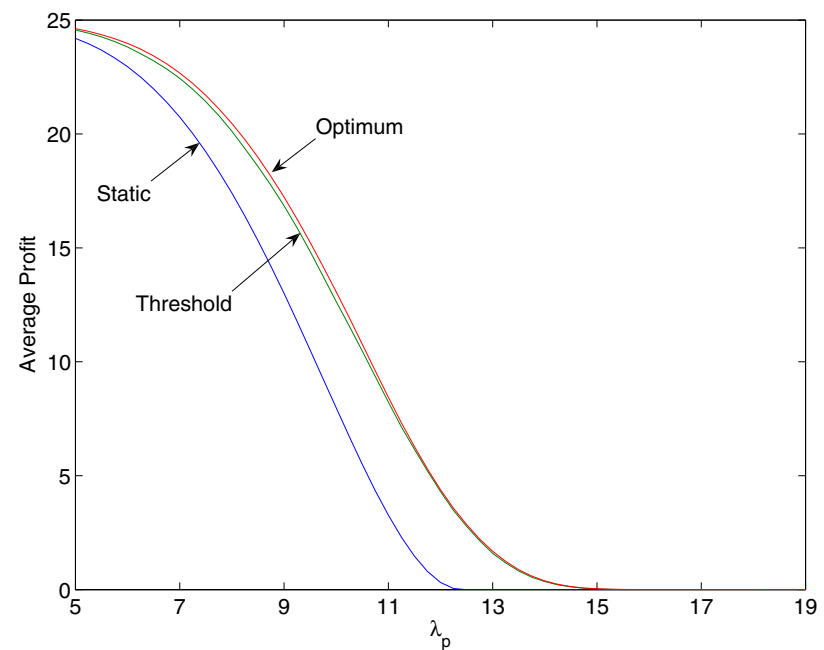

Fig. 3. Average profit vs primary load $\left(\lambda_{p}\right)$ for different pricing policies. System parameters: $C=20, K=100, \lambda_{s}(u)=(10-u)_{+}$and $\Delta u=$ $10^{-6}$.

are analogous to new and handover calls, respectively. It is shown in [13] that a threshold admission policy is the optimal solution for the MINOBJ problem and, thus, the same result applies to our setting. Note that the analogy is valid when $K>u$. If this is not the case, then the admission policy is obvious, namely, always admit SU calls.

Figure (3) compares the average profits achieved by the the optimal, static, and threshold policies for a linear demand function $\lambda_{s}(u)=(10-u)_{+}$(we explain in Section VI how to compute the optimal price and threshold). Figure (4) makes the same comparison for the following popular non-linear demand function [23]

$$
\lambda_{s}(u)=\left(A e^{-\gamma u^{2}}-\epsilon\right)_{+},
$$

where $A$ and $\gamma$ are scaling factors, and $\epsilon>0$ is a small constant introduced to enforce Assumption 3.1. Both figures show that threshold pricing performs close to optimal while static pricing performs significantly worse. Furthermore, we observe that beyond a certain value of $\lambda_{p}$, static pricing stops generating profit while threshold pricing continues doing so. This observation will be formalized in Section V-D.

\section{B. Properties of Threshold Pricing}

Having showed that threshold pricing is the optimal singleprice policy, we next derive an expression for the profit obtained with this policy, denoted by $R_{T}\left(\lambda_{s}\right)$. We denote profit function and blocking probabilities as a function of $\lambda_{s}$. This considerably simplifies notation in the rest of the paper. First, we define the following function which will be used extensively:

$$
Q\left(\lambda_{s}\right)=\lambda_{s} u\left(\lambda_{s}\right)
$$

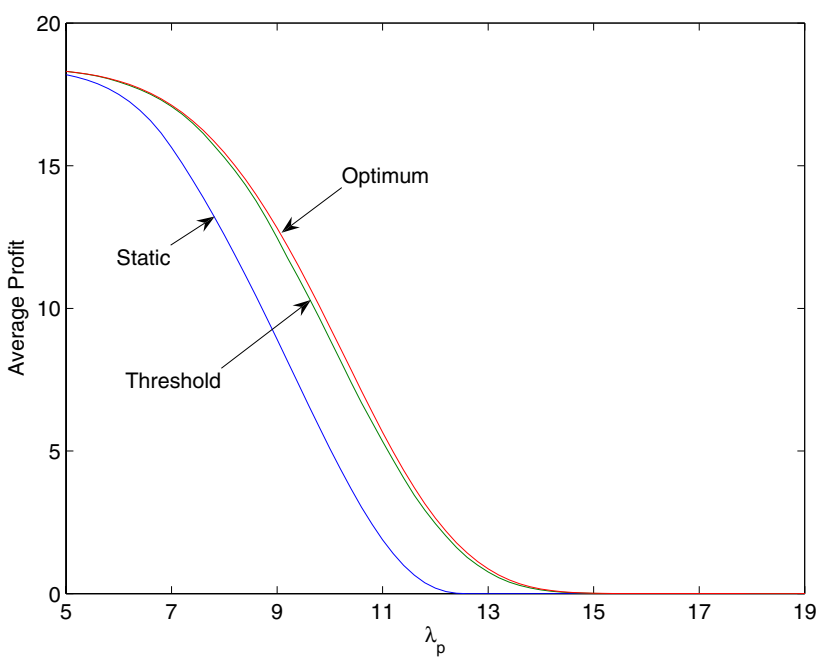

Fig. 4. Average profit vs primary load $\left(\lambda_{p}\right)$ for different pricing policies. System parameters: $C=20, K=100, \lambda_{s}(u)=\left(10 e^{-0.04 u^{2}}-0.1\right)_{+}$and $\Delta u=10^{-6}$.

where $u\left(\lambda_{s}\right)$ is the inverse function of $\lambda_{s}(u)$. Then, we compute the blocking probabilities for the PUs and SUs:

$$
\begin{aligned}
B_{P U}\left(\lambda_{s}, T\right) & =\pi_{C} \\
& =\frac{\frac{\left(\lambda_{s}+\lambda_{p}\right)^{T} \lambda_{p}^{C-T}}{C !}}{\sum_{n=0}^{T-1} \frac{\left(\lambda_{s}+\lambda_{p}\right)^{n}}{n !}+\left(\lambda_{s}+\lambda_{p}\right)^{T} \sum_{n=T}^{C} \frac{\lambda_{p}^{n-T}}{n !}} ; \\
B_{S U}\left(\lambda_{s}, T\right) & =\sum_{n=T}^{C} \pi_{n} \\
& =\frac{\left(\lambda_{s}+\lambda_{p}\right)^{T} \sum_{n=T}^{C} \frac{\lambda_{p}^{n-T}}{n !}}{\sum_{n=0}^{T-1} \frac{\left(\lambda_{s}+\lambda_{p}\right)^{n}}{n !}+\left(\lambda_{s}+\lambda_{p}\right)^{T} \sum_{n=T}^{C} \frac{\lambda_{p}^{n-T}}{n !}} .
\end{aligned}
$$

Note that, arrival rate until congestion level reaches $T$ channels is $\lambda_{s}+\lambda_{p}$ and just $\lambda_{p}$ afterwards.

Finally, we can provide an explicit expression for $R_{T}\left(\lambda_{s}\right)$ as follows:

$$
\begin{array}{r}
R_{T}\left(\lambda_{s}\right)=\left(1-B_{S U}\left(\lambda_{s}, T\right)\right) Q\left(\lambda_{s}\right)- \\
B_{P U}\left(\lambda_{s}, T\right) \lambda_{p} K \\
+E\left(\lambda_{p}, C\right) \lambda_{p} K .
\end{array}
$$

The first term in Eq. (12) is the revenue collected from SU calls. The second term is a result of the punishment due to blocked PU calls. The last term is the normalization term which is used to ensure that profit is zero when there are no SUs (see Eq. (3)).

Next, we derive an important property of the blocking probabilities $B_{P U}$ and $B_{S U}$, that will be exploited in the next section. Specifically, we show that the ratio of these blocking probabilities depends only on the PU's call arrival rate $\lambda_{p}$ and threshold $T$ but not on the price or the demand function of the SU.

Lemma 5.2: The ratio $\frac{B_{P U}\left(\lambda_{s}, T\right)}{B_{S U}\left(\lambda_{s}, T\right)}$ is independent of $u$ and 
Proof:

$$
\frac{B_{P U}\left(\lambda_{s}, T\right)}{B_{S U}\left(\lambda_{s}, T\right)}=\frac{\frac{\lambda_{p}^{C-T}}{C !}}{\sum_{n=T}^{C} \frac{\lambda_{p}^{n-T}}{n !}}
$$

which is independent of $u$ and $\lambda_{s}$.

\section{Unimodality of the Profit Function}

In this section, we show that for each given threshold $T$, the profit function of threshold pricing $R_{T}$ is unimodal in $u$ (a function is unimodal over a certain interval, if it has a single maximum over that interval). This result requires the following mild assumption on the demand function:

Assumption 5.3: The function $Q\left(\lambda_{s}\right)$ is concave i.e., $Q^{\prime \prime}\left(\lambda_{s}\right) \leq 0$ where the derivative is taken with respect to $\lambda_{s}$.

Assumption 5.3 is widely used in the literature [6,9] and is satisfied by several types of functions, such as linear and exponential decaying demand functions.

The proof of our theorem will be based on the following lemma, which is proved in the Appendix.

Lemma 5.4: For all $\lambda_{s}>0$,

$$
\frac{B_{S U}\left(\lambda_{s}, T\right)}{1-B_{S U}\left(\lambda_{s}, T\right)}>\frac{-B_{S U}^{\prime \prime}\left(\lambda_{s}, T\right)}{2 B_{S U}^{\prime}\left(\lambda_{s}, T\right)},
$$

where the derivatives are taken with respect to $\lambda_{s}$.

We can now state our theorem:

Theorem 5.5: For a fixed threshold $T$, the function $R_{T}$ is unimodal with respect to the price $u$ over the interval $\left[0, u_{\max }\right]$.

Proof: We will prove that $R_{T}\left(\lambda_{s}\right)$ is unimodal with respect to $\lambda_{s}$. Since by Assumption 3.1 the function $\lambda_{s}(u)$ is strictly decreasing, this will also prove the unimodality of $R_{T}$ with respect to $u$.

We refer to any value of $\lambda_{s}$ at which the derivative of $R_{T}\left(\lambda_{s}\right)$ is equal to zero as a critical point. We will denote such a point with $\lambda_{s}^{*}$, i.e., $R_{T}^{\prime}\left(\lambda_{s}^{*}\right)=0$. To prove the theorem, we will show that $R_{T}^{\prime \prime}\left(\lambda_{s}^{*}\right)<0$, for any $\lambda_{s}^{*}$. This means that there can be at most one critical point and it must be a maximum.

Let $X=\frac{B_{P U}\left(\lambda_{s}, T\right)}{B_{S U}\left(\lambda_{s}, T\right)}$ (recall Lemma 5.2). Then, we can rewrite the profit function and its first and second derivatives as follows:

$$
\begin{aligned}
R_{T}\left(\lambda_{s}\right)= & \left(1-B_{S U}\left(\lambda_{s}, T\right)\right) Q\left(\lambda_{s}\right) \\
- & X B_{S U}\left(\lambda_{s}, T\right) \lambda_{p} K+E\left(\lambda_{p}, C\right) \lambda_{p} K \\
R_{T}^{\prime}\left(\lambda_{s}\right)= & \left(1-B_{S U}\left(\lambda_{s}, T\right)\right) Q^{\prime}\left(\lambda_{s}\right) \\
& -B_{S U}^{\prime}\left(\lambda_{s}, T\right)\left(Q\left(\lambda_{s}\right)+X \lambda_{p} K\right) \\
R_{T}^{\prime \prime}\left(\lambda_{s}\right)= & \left(1-B_{S U}\left(\lambda_{s}, T\right)\right) Q^{\prime \prime}\left(\lambda_{s}\right) \\
& -B_{S U}^{\prime \prime}\left(\lambda_{s}, T\right)\left(Q\left(\lambda_{s}\right)+X \lambda_{p} K\right) \\
& -2 B_{S U}^{\prime}\left(\lambda_{s}, T\right) Q^{\prime}\left(\lambda_{s}\right)
\end{aligned}
$$

Since $R_{T}^{\prime}\left(\lambda_{s}^{*}\right)=0$, we obtain from Eq. (15):

$$
\frac{Q^{\prime}\left(\lambda_{s}^{*}\right)}{Q\left(\lambda_{s}^{*}\right)+X \lambda_{p} K}=\frac{B_{S U}^{\prime}\left(\lambda_{s}^{*}, T\right)}{1-B_{S U}\left(\lambda_{s}^{*}, T\right)}
$$

From Eq. (16) and Assumption 5.3, a sufficient condition for $R_{T}^{\prime \prime}\left(\lambda_{s}^{*}\right)<0$ is

$$
\frac{Q^{\prime}\left(\lambda_{s}^{*}\right)}{Q\left(\lambda_{s}^{*}\right)+X \lambda_{p} K} \geq \frac{-B_{S U}^{\prime \prime}\left(\lambda_{s}^{*}, T\right)}{2 B_{S U}^{\prime}\left(\lambda_{s}^{*}, T\right)},
$$

which holds true by Lemma 5.4 and Eq. (17).

\section{Characterization of the Profit Regions of Static and Thresh- old Pricing}

In this section, we characterize the profit regions of static and threshold pricing. Specifically, we are interested in determining the maximum value of $\lambda_{p}$, denoted by $\lambda_{p, \max }$, for which each of these policies still achieves a positive profit. The results in this section also require Assumption 5.3.

We prove that there exists a range of values of $\lambda_{p}$ (which can be very large) for which threshold pricing achieves a positive profit while static pricing does not. Remarkably, the value of $\lambda_{p, \max }$ for static pricing depends only on $u_{\max }$, but is independent of the demand function otherwise. Moreover, the lower bound we provide on threshold pricing profit region is also independent of the demand function.

We first establish the condition for which static pricing stops generating profit (i.e., blocks all SU calls).

Lemma 5.6: Optimal static pricing blocks all SU calls if and only if

$$
u_{\max } \leq\left(E\left(\lambda_{p}, C-1\right)-E\left(\lambda_{p}, C\right)\right) \lambda_{p} K .
$$

Proof: If all SU calls are blocked then the optimal SU arrival rate $\lambda_{s}^{*}$ is zero. We prove the lemma by showing that if condition (19) is satisfied then $\lambda_{s}^{*}=0$ and if it is not then $\lambda_{s}^{*} \neq 0$ (i.e., static pricing generates profit).

Next, we analyze the expression

$$
\left.\frac{\partial R_{C}\left(\lambda_{s}\right)}{\partial \lambda_{s}}\right|_{\lambda_{s}=0^{+}}
$$

where the notation $0^{+}$is used to mean that the derivative is taken to the right of 0 . If $\left.\frac{\partial R_{C}\left(\lambda_{s}\right)}{\partial \lambda_{s}}\right|_{\lambda_{s}=0^{+}}>0$ then there exists $\lambda_{s}>0$ that generates profit. (i.e., $\lambda_{s}^{*} \neq 0$ ).

In Theorem 5.5 we show that $R_{C}$ is unimodal with respect to $u$ and $\lambda_{s}$ under Assumption 5.3. Due to the unimodality of $R_{C}$, if $\left.\frac{\partial R_{C}\left(\lambda_{s}\right)}{\partial \lambda_{s}}\right|_{\lambda_{s}=0^{+}} \leq 0$ then $\lambda_{s}^{*}=0$.

Note that, in the case of static pricing

$$
B_{S U}\left(\lambda_{s}, C\right)=B_{P U}\left(\lambda_{s}, C\right)=E\left(\lambda_{s}+\lambda_{p}, C\right) .
$$

It can be verified that

$$
\begin{aligned}
\frac{\partial E\left(\lambda_{s}+\lambda_{p}, C\right)}{\partial \lambda_{s}} & =\left(1-E\left(\lambda_{s}+\lambda_{p}, C\right)\right) \\
\cdot & \left(E\left(\lambda_{s}+\lambda_{p}, C-1\right)-E\left(\lambda_{s}+\lambda_{p}, C\right)\right),
\end{aligned}
$$

and by using this equation we can evaluate $\frac{\partial R_{C}\left(\lambda_{s}\right)}{\partial \lambda_{s}}$ at $\lambda_{s}=$ $0^{+}$as

$$
\begin{aligned}
& \left.\frac{\partial R_{C}\left(\lambda_{s}\right)}{\partial \lambda_{s}}\right|_{\lambda_{s}=0^{+}}=\left(1-E\left(\lambda_{p}, C\right)\right) \\
& \quad \cdot\left(u_{\max }-\left(E\left(\lambda_{p}, C-1\right)-E\left(\lambda_{p}, C\right)\right) \lambda_{p} K\right) .
\end{aligned}
$$


Note that $E\left(\lambda_{p}, C\right)<1$. Therefore, the sign of Eq. (22) depends only on the last term.

An interesting corollary from this lemma is that if $K=0$ (i.e., there is no punishment), then static pricing policy will accept SUs for all values of $\lambda_{p}$, and hence achieves the maximum profit region. This result indicates that the nonoptimality of static pricing is due to both the presence of non-elastic PUs and punishments.

Next, we conduct a similar analysis for threshold pricing. We consider the case $T=1$. This will provide a lower bound on the profit region of threshold pricing. Note that if threshold pricing generates profit when $T=1$, it will also do so when the threshold is set to its optimal value.

Lemma 5.7: Threshold pricing with $T=1$ generates profit if and only if

$$
u_{\max } \geq E\left(\lambda_{p}, C\right) K .
$$

Proof: It can be shown that

$$
\begin{aligned}
& \frac{\partial B_{P U}\left(\lambda_{s}, 1\right)}{\partial \lambda_{s}}=\left(1-B_{S U}\left(\lambda_{s}, 1\right)\right) B_{P U}\left(\lambda_{s}, 1\right) \frac{1}{\lambda_{s}+\lambda_{p}}, \\
& \frac{\partial B_{S U}\left(\lambda_{s}, 1\right)}{\partial \lambda_{s}}=\left(1-B_{S U}\left(\lambda_{s}, 1\right)\right) B_{S U}\left(\lambda_{s}, 1\right) \frac{1}{\lambda_{s}+\lambda_{p}} .
\end{aligned}
$$

By using Eqs. (24) and (25), we can evaluate $\frac{\partial R_{1}\left(\lambda_{s}\right)}{\partial \lambda_{s}}$ at $\lambda_{s}=$ $0^{+}$as

$$
\begin{aligned}
\left.\frac{\partial R_{1}\left(\lambda_{s}\right)}{\partial \lambda_{s}}\right|_{\lambda_{s}=0^{+}}= & \left(1-B_{S U}(0,1)\right) \\
& \cdot\left(u_{\max }-B_{P U}(0,1) K\right) .
\end{aligned}
$$

Note that, $B_{P U}(0,1)=E\left(\lambda_{p}, C\right)$. The result follows based on arguments similar to those of the previous lemma.

It is a reasonable conjecture that maximum profit region for threshold pricing is archived when $T=1$. This is supported by our simulations.

We next show that the profit region of threshold pricing is larger than that of static pricing. We do so by showing that the RHS of Eq. (23) is larger than that of Eq. (19).

Lemma 5.8: For $C>1$ and $\lambda_{p}>0$

$$
\left(E\left(\lambda_{p}, C-1\right)-E\left(\lambda_{p}, C\right)\right) \lambda_{p}>E\left(\lambda_{p}, C\right) .
$$

Proof: Manipulating expressions we obtain that Eq. (27) holds if only if

$$
E\left(\lambda_{p}, C\right)>\frac{\lambda_{p}-C}{\lambda_{p}} \Leftrightarrow 1-E\left(\lambda_{p}, C\right)<\frac{C}{\lambda_{p}} .
$$

Note that at this point the claim is proved for $C>\lambda_{p}$. Further manipulation yields

$$
\begin{gathered}
1-E\left(\lambda_{p}, C\right)<\frac{1}{\lambda_{p}} \frac{\sum_{n=0}^{C-1} \frac{\lambda_{p}^{n}}{n !}}{\sum_{n=1}^{C} \frac{\lambda_{p}^{n-1}}{n !}} \\
=\frac{1}{\lambda_{p}} \frac{1+\sum_{n=1}^{C-1} \frac{\lambda_{p}^{n}}{n !}}{1+C^{-1} \sum_{n=2}^{C} \frac{\lambda_{p}^{n-1} C}{n !}} \\
<\frac{1}{\lambda_{p}} \frac{1+\sum_{n=1}^{C-1} \frac{\lambda_{p}^{n}}{n !}}{1+C^{-1} \sum_{n=1}^{C-1} \frac{\lambda_{p}^{n}}{n !}}<\frac{C}{\lambda_{p}} .
\end{gathered}
$$

\begin{tabular}{|c|c|c|c|c|}
\hline \multirow{2}{*}{$u_{\max }$} & \multicolumn{2}{|c|}{$C=20$} & \multicolumn{2}{c|}{$C=40$} \\
\cline { 2 - 5 } & $\lambda_{p, \max }^{S P}$ & $\lambda_{p, \max }^{T P}$ & $\lambda_{p, \max }^{S P}$ & $\lambda_{p, \max }^{T P}$ \\
\hline 10 & 12.4 & 17.6 & 28.6 & 38.8 \\
30 & 15.4 & 25.6 & 33.1 & 54.2 \\
50 & 18.2 & 38.2 & 37.2 & 78.1 \\
70 & 22.4 & 65.3 & 42.9 & 98.6 \\
\hline
\end{tabular}

TABLE I

MAXIMUM VALUES OF $\lambda_{p}$ FOR WHICH STATIC PRICING (SP) AND THRESHOLD PRICING (TP) GENERATE PROFITS FOR $\lambda_{s}(u)=\left(u_{\max }-u\right)+$ AND $K=100$.

Finally, we state our theorem which is a result of the previously stated lemmas.

Theorem 5.9: If Eq. (27) holds then for any demand function $\lambda_{s}(u)$ there exists values of $\lambda_{p}$ for which static pricing blocks all SU calls but threshold pricing does not.

Proof: By Lemma 5.8, there exist a $\lambda_{p}$ for which the following is true:

$\left(E\left(\lambda_{p}, C-1\right)-E\left(\lambda_{p}, C\right)\right) \lambda_{p} K \geq u_{\max }>E\left(\lambda_{p}, C\right) K$

and by Lemmas 5.6 and 5.7 the result follows.

Table I provides a comparison for linear demand function with different values of $u_{\max }$. We observe that the difference can be very large. For instance, for the case $C=20$ and $u_{\max }=70$, the value of $\lambda_{p, \max }$ for threshold pricing is almost three times larger than that for static pricing.

\section{EFFICIENT COMPUTATION OF THE OPTIMAL THRESHOLD AND PRICE}

In Section $\mathrm{V}-\mathrm{C}$ we showed that the profit function of threshold pricing is unimodal. This allows us to exploit efficient logarithmic search techniques, such as Fibonacci search, to find the optimum price for a given threshold.

Our numerical results in the previous section have showed that threshold pricing performs close to optimal and far better than static pricing. In this section, we show another benefit of threshold pricing, namely, low computational complexity. Specifically, we show that the optimal price $u^{*}$ and corresponding optimal threshold $T^{*}\left(u^{*}\right)$ can be computed using $O(C \log |\mathbb{U}|)$ iterations. This result is significant because the discretization step $\Delta u$ should be chosen very small in order to minimize the loss of profit due to price discretization.

We can further speed up the process by reducing the search range. The following lemma shows that the optimum price for threshold pricing is higher than or equal to $u_{\infty}$. Hence, we can restrict the search range for the optimum price to the interval $\left[u_{\infty}, u_{\max }\right]$.

Lemma 6.1: For any given threshold $T, u^{*}(T)>u_{\infty}$, where $u^{*}(T)$ is the optimum price when the threshold is set to $T$.

Proof: Let $u_{-}<u_{\infty}$. From Assumption 3.1 on the demand function, we know that $\lambda_{s}\left(u_{-}\right)>\lambda_{s}\left(u_{\infty}\right)$ and $Q\left(\lambda_{s}\left(u_{-}\right)\right)<Q\left(\lambda_{s}\left(u_{\infty}\right)\right)$. Moreover, for any 
$T$ we have $B_{S U}\left(\lambda_{s}\left(u_{\infty}\right), T\right)<B_{S U}\left(\lambda_{s}\left(u_{-}\right), T\right)$ and $B_{P U}\left(\lambda_{s}\left(u_{\infty}\right), T\right)<B_{P U}\left(\lambda_{s}\left(u_{-}\right), T\right)$. These inequalities lead to the following conclusion:

$$
\begin{aligned}
& \left(1-B_{S U}\left(\lambda_{s}\left(u_{-}\right), T\right)\right) Q\left(\lambda_{s}\left(u_{-}\right)\right)-B_{P U}\left(\lambda_{s}\left(u_{-}\right), T\right) \lambda_{p} K \\
& \quad<\left(1-B_{S U}\left(\lambda_{s}\left(u_{\infty}\right), T\right)\right) Q\left(\lambda_{s}\left(u_{\infty}\right)\right)-B_{P U}\left(\lambda_{s}\left(u_{\infty}\right), T\right) \lambda_{p} K .
\end{aligned}
$$

Thus, for any $T$ (including $T^{*}$ ) the profit decreases as prices go below $u_{\infty}$. Therefore, for any $T, u^{*}(T) \geq u_{\infty}$.

Next, we prove that optimal threshold is $T_{\infty} \leq T^{*} \leq C$ where

$$
T_{\infty}=\arg \max _{0 \leq T \leq C}\left(R_{T}\left(\lambda_{s}\left(u_{\infty}\right)\right)\right.
$$

i.e., $T_{\infty}$ is the optimal threshold when price is set to $u_{\infty}$. This statement enables us to reduce search interval for the optimum threshold. Thus speeding up the computation time of optimal threshold policy. For the proof we need the following lemma.

Lemma 6.2: Assume $\lambda_{s}$ and $u$ are independent variables. The optimal threshold $T^{*}$ is a non-increasing function of $\lambda_{s}$ assuming $u$ is fixed. It is also non-decreasing function of $u$ assuming $\lambda_{s}$ is fixed.

Proof: This lemma is obtained by applying Theorem 2 of [18] (see Section II). In order to apply results of [18] to our threshold model we assume that there are no walk-in users. We consider PUs and SUs as two different contract user classes for which prices are set to $K$ and $u$ and arrival rates are $\lambda_{p}$ and $\lambda_{s}$, respectively. Theorem 2 of [18] states that in such a setting if we increase $u$ ( $\lambda_{s}$ is fixed) the corresponding optimal threshold for SUs will increase. Moreover, if we reduce $\lambda_{s}$ (u is fixed) the threshold will increase again.

Now, we can state the theorem

Theorem 6.3: Optimal threshold is in the range

$$
T_{\infty} \leq T^{*} \leq C .
$$

Proof: Assume $u$ is increased in the amount of $\alpha$ units to $u+\alpha$. Let $\beta=\lambda_{s}(u)-\lambda_{s}(u+\alpha)$ be the corresponding decrease in demand. By using Lemma 6.2 we can claim

$$
T^{*}\left(\lambda_{s}, u\right) \leq T^{*}\left(\lambda_{s}, u+\alpha\right) \leq T^{*}\left(\lambda_{s}-\beta, u+\alpha\right)
$$

Eq. (33) means if we increase $u$, the corresponding optimal threshold will not decrease.

Since optimal price, $u^{*}$, is higher than $u_{\infty}$, we can conclude that $T_{\infty} \leq T^{*}$.

Using the above theorem and lemmas, we can thus compute $T^{*}$ and $u^{*}\left(T^{*}\right)$ very easily, namely, we first compute the optimal price $u^{*}(T)$ (within the range $\left[u_{\infty}, u_{\max }\right]$ ) for each threshold $T \in\left[T_{\infty}, C\right]$, using a logarithmic search procedure.

Then, we establish the optimal threshold

$$
T^{*}=\arg \max _{T_{\infty} \leq T \leq C} R_{T}\left(\lambda_{s}\left(u^{*}(T)\right)\right),
$$

with corresponding optimal price $u^{*}\left(T^{*}\right)$.

In Table II, we present a numerical comparison of the time required to compute the optimal threshold policy and optimal

\begin{tabular}{|c|c|c|c|c|}
\hline$C$ & $t_{\text {run }}^{\text {OP }} / t_{\text {run }}^{T P}$ & $R_{O P}$ & $R_{T P}$ & $R_{S P}$ \\
\hline 250 & 5.9 & 3.8 & 3.1 & 0 \\
500 & 11.1 & 42.1 & 39.7 & 15.0 \\
750 & 18.7 & 111.6 & 108.4 & 75.5 \\
1000 & 27.7 & 188.6 & 185.7 & 155.3 \\
\hline
\end{tabular}

TABLE II

REVENUES OF OPTIMAL DYNAMIC PRICING (OP), TP, AND SP AND RATIO OF RUN TIMES OF OP AND TP. SYSTEM PARAMETERS: $\lambda_{p}=\frac{9}{10} C$, $K=100, \lambda_{s}(u)=\frac{C}{250}\left(10 e^{-\left(\frac{u}{5}-1\right)^{2}}-10^{-1}\right)+$ FOR $u \geq 5$, AND
$\Delta u=10^{-6}$.

dynamic policy. For computation of optimal dynamic policy policy iteration is used due to reasons mentioned in section IV. Moreover, the policy iteration procedure is also speeded up by taking advantage of unimodality. Both algorithms were developed in MATLAB and run on a Pentium M 1.7GHz PC. These numerical results demonstrate the practical importance of the optimization developed in this section.

\section{CONCLUding REMARKS}

In this paper we have investigated the problem of devising efficient pricing policies for secondary spectrum usage. Such policies are essential in order to minimize the loss of efficiency in transactions, which could limit the granularity and thereby liquidity of secondary spectrum markets.

Specifically, we have formalized the problem of profit maximization for the usage of wireless spectrum in the presence of both primary and secondary users (PUs and SUs). We have provided a stochastic dynamic programming formulation of the problem and shown how to derive the optimal stationary pricing policy using policy iteration or relative value iteration.

A drawback of the optimal dynamic policy is to charge SUs different prices over time, depending on the channel occupancy. This makes the cost of spectrum access much less predictable and could potentially reduce demand. Thus, we have investigated the design of simple, yet efficient, singleprice policies. We have provided numerical and analytical evidences that static pricing policies do not perform well in such settings (in contrast to settings where all the users are elastic). On the other hand, we have proven that deterministic threshold pricing achieves the optimal profit amongst all the single-price policies and performs close to global optimal pricing for a variety of demand functions. Under certain reasonable assumptions on the demand function, we have also proven that the profit function of threshold pricing is unimodal in the price. We have also showed that the optimal threshold lies within a specific interval. By taking advantage of these properties we showed that the optimal threshold policy is computationally more efficient than the optimal dynamic policy.

Lastly, we have characterized the profit regions of static and threshold pricing, as a function of the arrival rate of PUs. We have shown that these regions critically depend on the support of the demand function and, in particular, the parameter $u_{\max }$, but not on the specific form of the demand function. We have 
shown that threshold pricing is capable to achieve a much larger profit region. The numerical results also seem to indicate that the profit region of threshold pricing is identical to that of optimal pricing. However, a formal proof of this conjecture still remains an open problem.

\section{ApPendiX: Proof of Lemma 5.4}

It can be shown that

$$
\frac{\partial}{\partial \lambda_{s}}\left(-\ln \left(1-B_{S U}\left(\lambda_{s}, T\right)\right)\right)=\frac{B_{S U}^{\prime}\left(\lambda_{s}, T\right)}{1-B_{S U}\left(\lambda_{s}, T\right)}
$$

and

$$
\frac{\partial}{\partial \lambda_{s}}\left(\frac{1}{2} \ln \left(-B_{S U}^{\prime}\left(\lambda_{s}, T\right)\right)\right)=-\frac{B_{S U}^{\prime \prime}\left(\lambda_{s}, T\right)}{2 B_{S U}^{\prime}\left(\lambda_{s}, T\right)}
$$

from which it follows that

$$
\begin{aligned}
& \frac{B_{S U}\left(\lambda_{s}, T\right)}{1-B_{S U}\left(\lambda_{s}, T\right)}-\frac{-B_{S U}^{\prime \prime}\left(\lambda_{s}, T\right)}{2 B_{S U}^{\prime}\left(\lambda_{s}, T\right)}>0 \\
& \quad \Leftrightarrow \frac{1}{2} \frac{\partial}{\partial \lambda_{s}}\left(\ln \left(\frac{B_{S U}^{\prime}\left(\lambda_{s}, T\right)}{\left(1-B_{S U}\left(\lambda_{s}, T\right)\right)^{2}}\right)\right)>0 .
\end{aligned}
$$

Since the $\ln (\cdot)$ function is strictly increasing, we have

$$
\begin{aligned}
\frac{B_{S U}\left(\lambda_{s}, T\right)}{1-B_{S U}\left(\lambda_{s}, T\right)}-\frac{-B_{S U}^{\prime \prime}\left(\lambda_{s}, T\right)}{2 B_{S U}^{\prime}\left(\lambda_{s}, T\right)}>0 \\
\Leftrightarrow \frac{\partial}{\partial \lambda_{s}}\left(\frac{B_{S U}^{\prime}\left(\lambda_{s}, T\right)}{\left(1-B_{S U}\left(\lambda_{s}, T\right)\right)^{2}}\right)>0 .
\end{aligned}
$$

In [9] it is shown that inequality (39) is satisfied for an $M / M / T / T$ queueing system when $B_{S U}\left(\lambda_{s}, T\right)$ is replaced by $E\left(\lambda_{s}+\lambda_{p}, T\right)$ (the blocking probability in an $M / M / T / T$ queue). This inequality also holds for our system since

$$
\left.\frac{B_{S U}^{\prime}\left(\lambda_{s}, T\right)}{\left(1-B_{S U}\left(\lambda_{s}, T\right)\right)^{2}}\right)=Y\left(\frac{E^{\prime}\left(\lambda_{s}+\lambda_{p}, T\right)}{\left(1-E\left(\lambda_{s}+\lambda_{p}, T\right)\right)^{2}}\right)
$$

where $Y=T !\left(\frac{1}{T !}+\frac{\lambda_{p}}{(T+1) !}+\ldots+\frac{\lambda_{p}^{C-T}}{C !}\right)$. and $Y>0$. Thus, we have

$$
\frac{\partial}{\partial \lambda_{s}}\left(\frac{B_{S U}^{\prime}\left(\lambda_{s}, T\right)}{\left(1-B_{S U}\left(\lambda_{s}, T\right)\right)^{2}}\right)>0
$$

which proves the lemma.

\section{REFERENCES}

[1] "The Federal Communications Commission, Secondary Markets Initiative." http://wireless.fcc.gov/licensing/secondarymarkets/.

[2] "Radio Spectrum Policy Group." http://rspg.groups.eu.int/.

[3] R. Dewenter and J. Haucap, "Incentives to license mobile virtual network operators (MVNOs)," in Proc. 34th Research Conference on Communication, Information and Internet Policy, Oct. 2006.

[4] M. Lev-Ram, "As mobile ESPN falls, a Helio rises." http://money.cnn.com/2006/10/17/magazines/business2/ espnmobile_mvno.biz2/index.htm. CNN Business 2.0 Magazine.

[5] Z. Ezziane, "Charging and pricing challenges for 3G systems," IEEE Communications Surveys and Tutorials, vol. 7, pp. 58-68, Fourth Quarter 2005.

[6] I. C. Paschalidis and J. N. Tsitsiklis, "Congestion-dependent pricing of network services," IEEE/ACM Transactions on Networking, vol. 8 , pp. 171-184, April 2000

[7] I. C. Paschalidis and Y. Liu, "Pricing in multiservice loss networks: static pricing, asymptotic optimality, and demand substitution effects," IEEE/ACM Transactions on Networking, vol. 10, pp. 425-438, June 2002.
[8] X. Lin and N. Shroff, "Simplification of network dynamics in large systems," IEEE/ACM Transactions on Networking, vol. 13, pp. 813826, August 2005.

[9] S. Ziya, H. Ayhan, and R. Foley, "Optimal prices for finite capacity queueing systems,” Operations Research Letters, vol. 34, pp. 214-218, March 2006.

[10] A. Al-Daoud, M. Alanyali, and D. Starobinski, "Secondary pricing of spectrum in cellular CDMA networks," in IEEE DySPAN, 2007.

[11] G. Haring, R. Marie, R. Puigjaner, and K. Trivedi, "Loss formulas and their application to optimization for cellular networks," IEEE Transactions on Vehicular Technology, vol. 50, pp. 654-673, May 2001.

[12] S.-H. Oh and D.-W. Tcha, "Prioritized channel assignment in a cellular radio network," IEEE Transactions on Communications, vol. 40, pp. 1259-1269, July.

[13] R. Ramjee, R. Nagarajan, and D. F. Towsley, "On optimal call admission control in cellular networks," in INFOCOM, pp. 43-50, 1996.

[14] B. Miller, "A queueing reward system with several customer classes," Management Science, vol. 16, no. 3, 1969.

[15] J. Hou, J. Yang, and S. Papavassiliou, "Integration of pricing with call admission control to meet qos requirements in cellular networks," IEEE Transactions on Parallel \& Distributed Systems, vol. 13, no. 9, pp. 898910, 2002.

[16] I.-R. Chen, O. Yilmaz, and I.-L. Yen, "Admission control algorithms for revenue optimization with qos guarantees in mobile wireless networks," Wireless Personal Communication, vol. 38, no. 3, pp. 357-376, 2006.

[17] S.-L. Hew and L. White, "Optimal integrated call admission control and dynamic pricing with handoffs and price-affected arrivals," in Communications, 2005 Asia-Pacific Conf. on Communications, pp. 396400.

[18] N. Gans and S. Savin, "Pricing and capacity rationing for rentals with uncertain durations," Management Science, vol. 53, March 2007.

[19] S. Ross, Introduction to Stochastic Dynamic Programming. Academic Press, 1983

[20] D. Bertsekas, Dynamic Programming and Optimal Control. Athena Scientific, 2005.

[21] V. D. Blondel and J. N. Tsitsiklis, "A survey of computational complexity results in systems and control," Automatica, vol. 36, no. 9, pp. 12491274, 2000.

[22] H. Mutlu, M. Alanyali, and D. Starobinski, "Spot pricing of secondary spectrum usage in wireless cellular networks." Boston University, Center for Information and Systems Engineering, Technical Report, 2008.

[23] P. Fishburn and A. Odlyzko, "Dynamic behavior of differential pricing and quality of service options for the internet," Decision Support Systems, vol. 23, pp. 123-136, March 2000. 